\title{
Conformational Dynamics of Asparagine at Coiled-Coil Interfaces
}

\author{
Franziska Thomas, ${ }^{*}{ }^{\dagger, \S}$ Ai Niitsu, ${ }^{\dagger}$ Alain Oregioni, ${ }^{\|}$Gail J. Bartlett, ${ }^{\dagger}$ and Derek N. Woolfson ${ }^{*},,^{\dagger}, \perp \odot$ \\ †School of Chemistry, University of Bristol, Cantock’s Close, Bristol BS8 1TS, U.K. \\ ${ }^{\ddagger}$ School of Biochemistry, University of Bristol, Medical Sciences Building, University Walk, Bristol BS8 1TD, U.K. \\ ${ }^{\S}$ Institute for Organic and Biomolecular Chemistry, Georg-August-Universität Göttingen, Tammannstraße 2, 37077 Göttingen, \\ Germany \\ ${ }$ MRC Biomedical NMR Centre, The Francis Crick Institute, 1 Midland Road, London NW1 1AT, U.K. \\ ${ }^{\perp}$ BrisSynBio, University of Bristol, Life Sciences Building, Tyndall Avenue, Bristol BS8 1TQ U.K.
}

\section{Supporting Information}

\begin{abstract}
Coiled coils (CCs) are among the best-understood protein folds. Nonetheless, there are gaps in our knowledge of CCs. Notably, CCs are likely to be structurally more dynamic than often considered. Here, we explore this in an abundant class of CCs, parallel dimers, focusing on polar asparagine (Asn) residues in the hydrophobic interface. It is well documented that such inclusions discriminate between different CC oligomers, which has been rationalized in terms of whether the Asn can make side-chain hydrogen bonds. Analysis of parallel CC dimers in the Protein Data Bank reveals a variety of Asn side-chain conformations, but not all of these make the expected inter-side-chain hydrogen bond. We probe the structure and dynamics of a de novo-designed coiled-coil homodimer, CC-Di, by multidimensional nuclear magnetic resonance spectroscopy, including model-free dynamical analysis and relaxation-dispersion experiments. We



find dynamic exchange on the millisecond time scale between Asn conformers with the side chains pointing into and out of the core. We perform molecular dynamics simulations that are consistent with this, revealing that the side chains are highly dynamic, exchanging between hydrogen-bonded-paired conformations in picoseconds to nanoseconds. Combined, our data present a more dynamic view for Asn at CC interfaces. Although inter-side-chain hydrogen bonding states are the most abundant, Asn is not always buried or engaged in such interactions. Because interfacial Asn residues are key design features for modulating CC stability and recognition, these further insights into how they are accommodated within CC structures will aid their predictive modeling, engineering, and design.
\end{abstract}

\begin{abstract}
oiled coils (CCs) are some of the most abundant protein folds in nature. ${ }^{1}$ They play key roles in many biological processes, directing and stabilizing protein structures and protein-protein interactions. Over the past three decades, short CCs (20-40 amino acids long) have become favored models for studying protein folding and protein-protein interactions. As a result, CCs are one of the best understood protein-folding motifs available. ${ }^{1,2}$ In turn, this has led to them being used widely in peptide and protein design and engineering, and for applications in materials science, biotechnology, synthetic biology, and biomedicine. ${ }^{1-4}$ While sequencestructure relationships have been discerned, this does not mean that these are understood in physicochemical terms at the atomistic and molecular levels.

In CCs, two or more $\alpha$-helices combine to form bundles with a left-handed supercoil. Underlying this is a regular seven-residue or heptad-repeat sequence of hydrophobic $(\boldsymbol{h})$ and polar $(\boldsymbol{p})$ residues, hpphppp, commonly denoted abcdefg. ${ }^{2,5}$ When configured into an $\alpha$-helix, this pattern brings together the $a$ and $\boldsymbol{d}$ positions. The resulting hydrophobic face drives association of multiple helices to form the bundles. These
\end{abstract}

helix-helix interfaces are cemented by intimate side-chain interactions termed knobs-into-holes $(\mathrm{KIH})$ packing. ${ }^{6}$

Despite this apparent simplicity, CCs show a diversity of oligomeric states, the helices can be parallel or antiparallel, and the complexes can be homo- or heteromeric. In nature, CCs are mostly dimeric, trimeric, and tetrameric, although pentamers and higher-order oligomers are known both in nature and through design. ${ }^{2,5,7}$ The influence of the type of the residues at the $\boldsymbol{a}$ and $\boldsymbol{d}$ positions on the oligomeric state has been studied systematically in the GCN4 leucine-zipper peptide (GCN4-p1). ${ }^{8}$ In detail, isoleucine (Ile) at position $\boldsymbol{a}$ and leucine (Leu) at position $\boldsymbol{d}$ guide association to parallel CC dimers; having Ile at positions $a$ and $\boldsymbol{d}$ results in trimers, and Leu at position $\boldsymbol{a}$ and Ile at position $\boldsymbol{d}$ are characteristic of tetrameric assemblies.

Charged amino acids at the $\boldsymbol{e}$ and $\boldsymbol{g}$ positions, notably glutamic acid (Glu) and lysine (Lys), also play roles in CC structure and stability. These residues can direct CC folding, parallel or

Received: August 29, 2017

Revised: October 25, 2017

Published: November 22, 2017 
antiparallel helix orientation, and homotypic versus heterotypic assembly. $^{9-14}$

Despite the importance of $h p$ patterns, polar residues occur surprisingly frequently at positions $\boldsymbol{a}$ and $\boldsymbol{d}$, accounting for $\approx 25 \%$ of all residues in the CC+ database of CCs culled from the Protein Data Bank (PDB). ${ }^{15,16}$ Although they are thermodynamically destabilizing, it has become evident that these residues play important roles in the specification of the $\mathrm{CC}$ oligomeric state and orientation..$^{10,13,17-21}$ Indeed, such residues are often highly conserved through evolution. ${ }^{22}$

Asparagine (Asn) is among the most abundant of these polar inclusions. ${ }^{23}$ For example, in the trimeric CC domains of the autotransporter adhesins, conserved Asn residues occur at position $\boldsymbol{d}^{24} \mathrm{X}$-ray crystal structures of these motifs show that these side chains are often associated with sequestered halide anions in the core. More commonly, however, buried Asn residues occur at the $\boldsymbol{a}$ sites of CC dimers, for example, the bZip transcription factors, ${ }^{25,26}$ where the residues are often required for dimer specificity. ${ }^{8,27,28}$ Indeed, Asn-at- $\boldsymbol{a}$ is now almost an obligatory design feature in the specification of completely de novo-designed homo- and heterodimeric CCs, and in the construction of orthogonal CC pairs. ${ }^{13,17,29-31}$ However, as a cautionary note and to add additional nuance to this, the Asn-at- $\boldsymbol{a}$ has to be located centrally to specify oligomer state; otherwise, alternative and unintended CC assemblies can be observed. ${ }^{32}$

These sequence-structure relationships present rules for the de novo design of CCs. On this basis, we and others have designed and characterized sets of homomeric and heteromeric coiled coils, which have been used in synthetic biology and materials design. ${ }^{12,13,17,33}$ However, the demands on de novo CCs through these and advancing applications are growing; notably, larger sets of orthogonal heterodimers are required for more complex CC assemblies, $^{34,35}$ and structures beyond simple dimers to tetramers are being designed. ${ }^{7,36}$ As a consequence, more sequence-structure relationships are needed, and we need to develop a deeper understanding of those that we have.

An important aspect of this will be to understand the dynamics of CCs, especially of residues at the CC interfaces, and how these contribute to CC specification. Analyses of crystal structures alone will not be sufficient as these give only one or a small number of the many possible CC conformations. The controversial discussion of whether interhelical electrostatic interactions in CC dimers are stabilizing provides an example of this. While X-ray crystal structures anticipate salt-bridge formation at CC interfaces, solution-phase nuclear magnetic resonance (NMR) gives a more complex view and generally identifies fewer salt bridges than expected. ${ }^{37-41}$ In GCN4-p1, acidic residues in these pairs make little contribution to dimer stability, but certain basic residues do contribute favorably. ${ }^{42,43}$ Nonetheless, $\mathrm{pH}$ titrations removing any formal electrostatic interactions result in subtle changes in the overall CC structure and supercoil. ${ }^{44}$

For buried Asn residues, hydrogen bonding between the amide side chains is observed in X-ray crystal structures ${ }^{25}$ and is commonly assumed to offset the destabilization of burying these functional groups. As with salt-bridge interactions, however, these interactions are not straightforward and are more likely highly dynamic. Indeed, the aforementioned $\mathrm{pH}$ titration of GCN4-p1 reveals a range of Asn side-chain conformations. ${ }^{44}$ Related to this, the native dimeric state of GCN4-p1, which has a central Asn-at-a, forms trimers under certain conditions. ${ }^{45}$ In this state, the Asn side chains point out from the hydrophobic core and water fills the space left behind. This dimer-trimer ambiguity is accentuated by mutating Asn to Gln in the GCN4 system. ${ }^{28}$ These conformational dynamics in GCN4-p1 might be extreme, but they are not unique. NMR spectroscopy focusing on core Asn residues of a disulfide-linked Jun homodimer confirms hydrogen-bonded Asn side chains but suggests fast exchange between two different asymmetric hydrogen bonds. ${ }^{46}$

Because of the importance of the buried Asn residues in CC assembly, recognition, and design, it is imperative to completely understand the dynamics and interactions of these inclusions. This would provide clarity about the mechanism of dimer specification by Asn in CC dimers and facilitate the manipulation of these interactions to redesign and design CC interfaces.

Here we shed light on Asn inclusions through detailed analysis, experiments, and modeling of a de novo-designed parallel CC homodimer, CC-Di. ${ }^{17} \mathrm{CC}-\mathrm{Di}$ has four heptads with Asn at the $a$ site of the third. This is highly specifying for dimer, as mutation to the canonical Ile gives a trimer. ${ }^{17}$ The X-ray crystal structure of the dimer shows that the core Asn residues are dynamic with multiple Asn-Asn paired conformations present. We combine analyses of Asn residues buried in dimeric CC interfaces of the $\mathrm{PDB}$ and two- to four-dimensional NMR experiments to probe the dynamics of Asn-Asn interaction directly. These reveal multiple paired Asn-Asn conformations. While the major conformers are the hydrogen-bonded pairs, other states exist in which the Asn side chains point out from the hydrophobic core and do not interact with each other. Finally, we perform molecular dynamics (MD) studies to visualize a mechanism for this exchange process.

\section{MATERIALS AND METHODS}

General. Fmoc-protected amino acids, DIC and HBTU, and peptide grade dimethylformamide were purchased from AGTC Bioproducts (Hessle, U.K.). H-Rink Amide-Chemmatrix resin was acquired from PCAS BioMatrix Inc. (Saint-Jean-surRichelieu, QC). ${ }^{15} \mathrm{~N}$-labeled amino acids Fmoc-Asn(Trt)-OH and Fmoc-Leu-OH were obtained from Sigma-Aldrich. H-Leu$\mathrm{OH}$, which was labeled with ${ }^{13} \mathrm{C}$ at the $\alpha$-carbon, was acquired from Campro Scientific (Berlin, Germany) and subsequently Fmoc-protected following standard procedures. All other chemicals were purchased form Fisher Scientific (Loughborough, U.K.). Water was purified with a Synergy ultraviolet (UV) water purification system from Millipore. Peptide concentrations were determined by UV absorbance $\left[\lambda_{280}(\operatorname{Trp})\right.$ $\left.=5690 \mathrm{~mol}^{-1} \mathrm{~cm}^{-1} ; \lambda_{280}(\mathrm{Tyr})=1280 \mathrm{~mol}^{-1} \mathrm{~cm}^{-1}\right]$ using a NanoDrop 2000 spectrophotometer from Thermo Scientific.

Peptide Synthesis. The peptide amides were synthesized on an H-Rink Amide-Chemmatrix resin on a $0.1 \mathrm{mmol}$ scale on a CEM microwave-assisted synthesizer. The synthesis was conducted via a standard Fmoc protocol using a DIC/HOBt coupling reagent mixture. N-Acetylation of the peptides was performed by using acetic acid anhydride and pyridine (1:9). Acidic cleavage from the resin was achieved by a treatment of the resin with a mixture of trifluoroacetic acid (TFA), triisopropylsilane, and water (90:5:5, $3 \mathrm{~h})$. The resin was extracted with additional TFA $(5 \mathrm{~mL})$, and the combined extracts were concentrated to a third of the initial volume under a flow of nitrogen. The crude peptide was then precipitated in cold diethyl ether $(40 \mathrm{~mL})$ and isolated by centrifugation and decantation of the ether. The precipitate was redissolved in $5 \mathrm{~mL}$ of a 1:1 mixture of acetonitrile and water and then freeze-dried to give a fine white solid.

Peptide Purification. Peptides were purified by reversephase high-performance liquid chromatography (HPLC) using a 
JASCO chromatography system and a Kromatek C18HQsil column $(150 \mathrm{~mm} \times 10 \mathrm{~mm})$ on which a gradient of water and acetonitrile (buffer A consisted of water and 0.1\% TFA, and buffer $B$ consisted of acetonitrile and $0.1 \%$ TFA) run from 30 to $60 \%$ B over 30 min was applied.

Peptide Characterization. The peptides were characterized by mass spectrometry on a Bruker Daltonics UltrafleXtreme matrix-assisted desorption ionization time-of-flight mass spectrometer operating in positive-ion reflector mode [matrix of $\alpha$ cyano-4-hydroxycinnamic acid (CHCA), external calibration]. High-resolution mass spectrometry was performed on a Waters Synapt G2-S nano-ESI-IMS-TOF mass spectrometer. Analytical HPLC measurements were performed using a JASCO chromatography system and a Phenomenex Prodigy ODS-3 column $(5 \mu \mathrm{m}, 4.6 \mathrm{~mm} \times 100 \mathrm{~mm})$. For peptide characterization, a linear gradient of water and acetonitrile (buffer A consisted of water and $0.1 \%$ TFA, and buffer B consisted of acetonitrile and $0.1 \%$ TFA) run from 20 to $80 \%$ over $20 \mathrm{~min}$ was used. Chromatograms were monitored at wavelengths of 220 and 280 $\mathrm{nm}$.

NMR Measurements. The unlabeled and labeled CC-Di samples were made at a total peptide concentration of $2 \mathrm{mM}$ in a phosphate buffer [ $8.2 \mathrm{mM}$ sodium phosphate, $1.8 \mathrm{mM}$ potassium phosphate, $68 \mathrm{mM}$ sodium chloride, and $1.4 \mathrm{mM}$ potassium chloride ( $\mathrm{pH}$ adjusted to 7.0 )] containing $10 \% \mathrm{D}_{2} \mathrm{O}$ at 20 or 40 ${ }^{\circ} \mathrm{C}$. The NMR data were acquired on Bruker Avance and Avance III spectrometers at $600 \mathrm{MHz}$ (TCI Cryoprobe) and Bruker Avance III HD spectrometers at $700 \mathrm{MHz}$ (QCI Cryoprobe) and $800 \mathrm{MHz}$ (TCI Cryoprobe). Primary assignments and structure calculations for CC-Di were obtained from the unlabeled sample using proton two-dimensional (2D) TOCSY (60 ms mixing time), 2D NOESY ( 80 and $150 \mathrm{~ms}$ mixing times ), 2D COSY, and ${ }^{15} \mathrm{~N}$-edited SOFAST-HMQC ${ }^{47}$ at $600 \mathrm{MHz}$.

A selectively labeled $\left[{ }^{15} \mathrm{~N}\right] \mathrm{Leu}$ and Asn sample was used at 600 and $700 \mathrm{MHz}$ to study the dynamic properties of CC-Di. A model-free approach was used on the data from ${ }^{15} \mathrm{~N}$-edited $T_{1}$, $T_{2}$, and ${ }^{15} \mathrm{~N}{ }^{1} \mathrm{NOE}$ of the backbone residues. ${ }^{48}$ The conformational exchange detected on the labeled Asn amide side chains was studied by ${ }^{15} \mathrm{~N}$-edited relaxation-dispersion experiments. ${ }^{49}$

To study the connectivity of CC-Di, an equimolar mixture of 1 $\mathrm{mM}$ selectively ${ }^{15} \mathrm{NN} \varepsilon$-labeled Asn 17 sample and $1 \mathrm{mM}$ selectively ${ }^{13} \mathrm{C} \alpha$-labeled Leu13 sample was prepared, yielding a $0.5 \mathrm{mM}$ abundance of CC-Di with one coil being ${ }^{15} \mathrm{~N}$-labeled Asn and the other ${ }^{13} \mathrm{C}$-labeled Leu. The trans-coil connectivity was checked through a four-dimensional (4D) ${ }^{13} \mathrm{C}$-edited-HSQCNOE- ${ }^{15} \mathrm{~N}$-edited-HMQC ${ }^{50}$ with a $120 \mathrm{~ms}$ NOESY mixing time at $800 \mathrm{MHz}$. Because of the selective labeling limiting the number of peaks observed, only the proton-proton $2 \mathrm{D}$ plane was necessary.

The same sequence was used in a one-dimensional (1D) form to record an NOE buildup to gain insight into the crossrelaxation of the labeled spins. The NOE buildup rate is proportional to cross-relaxation $\sigma$ from a spin $S$ to a spin I, following:

$$
\frac{\mathrm{d} I}{\mathrm{~d} t}=\sigma_{\mathrm{IS}} S_{z 0}
$$

where $S_{z 0}$ is the starting magnetization of spin S.

In turn, the cross-relaxation is linked to distance $R_{\mathrm{IS}}$ between spins I and S: ${ }^{51}$

$$
R_{\mathrm{IS}}=\left(\frac{59.94 \tau_{\mathrm{C}}}{\sigma_{\mathrm{IS}}}\right)^{1 / 6}
$$

where $\tau_{\mathrm{C}}$ is the molecular correlation time in nanoseconds giving $R_{\mathrm{IS}}$ in angstroms.

One problem with measuring the cross-relaxation is a lack of normalization of the NMR signals. Here, we use the knowledge of the $\mathrm{N} 17_{\mathrm{NH}}-\mathrm{L} 13_{\mathrm{C} \alpha \mathrm{H}}$ proton distance from the crystal structure $\left(R_{\mathrm{N} 17-\mathrm{L} 13}^{\mathrm{X}}\right)$. From eq 2 , we obtain

$$
\sigma_{\mathrm{NH}}=\frac{59.94 \tau_{\mathrm{C}}}{\left(R_{\mathrm{N} 17-\mathrm{L} 13}^{\mathrm{X}}\right)^{6}}
$$

where $\sigma_{\mathrm{NH}}$ is the cross-relaxation between the N17 amide proton of one strand and the L13 $\alpha$-proton of the other strand of the dimer and $\tau_{\mathrm{C}}$ the global correlation time of the dimer. Replacing $\sigma$ in eq 1 allows us to eliminate $S_{z 0}$ (representing the $\mathrm{L} 13 \mathrm{H} \alpha$ starting magnetization):

$$
S_{z 0}=\frac{\mathrm{d} I_{\mathrm{NH}}}{\mathrm{d} t} \frac{\left(R_{\mathrm{N} 17-\mathrm{L} 13}^{\mathrm{X}}\right)^{6}}{59.94 \tau_{\mathrm{C}}}
$$

where $\mathrm{d} I_{\mathrm{NH}} / \mathrm{d} t$ is the NOE buildup curve of the N17 amide proton. Because the starting point of the magnetization for the NOE transfer to the side chains of $\mathrm{N} 17$ was also $\mathrm{L} 13 \mathrm{H} \alpha$, we can substitute $S_{z 0}$ in the buildup calculations, giving us a way to evaluate the cross-relaxation and hence the distances to the side chains:

$$
\sigma_{\mathrm{SC}}=\frac{\frac{\mathrm{d} I_{\mathrm{SC}}}{\mathrm{d} t}}{\frac{\mathrm{d} I_{\mathrm{NH}}}{\mathrm{d} t}} \frac{59.94 \tau_{\mathrm{C}}}{\left(R_{\mathrm{N} 17-\mathrm{L} 13}^{\mathrm{X}}\right)^{6}}
$$

where $\sigma_{\mathrm{SC}}$ is the cross-relaxation between the N17 side-chain protons of one strand and the L13 $\alpha$-proton of the other strand and $\tau_{\mathrm{C}}$ the global correlation time of the dimer. The ratio of the buildup curves can be substituted for the ratio of the slopes of the buildup curves calculated with a normalization to the $\mathrm{NH}$ peaks. This allows a distance to be calculated once it is placed back into eq 2:

$$
R_{\mathrm{SC}}=R_{\mathrm{N} 17-\mathrm{L} 13}^{\mathrm{X}}\left(\frac{\frac{\mathrm{d} \mathrm{I}_{\mathrm{NH}}}{\mathrm{d} t}}{\frac{\mathrm{d} I_{\mathrm{SC}}}{\mathrm{d} t}}\right)^{1 / 6}
$$

Equations 1-6 are set for a standard setup of proton-proton interaction. In our case, we know that the side chains are in exchange. However, because the exchange rate is slow to intermediate (only one peak is seen) and likely to be around 20 $\mathrm{s}^{-1}$, substantially more than the $R_{1}$ values of any backbone or side-chain residues, the dependence of exchange on the measured $\left(R_{1}\right.$, NOE buildup) or calculated $\left(\sigma_{\mathrm{IS}}\right.$ or $\left.R_{\mathrm{IS}}\right)$ values is averaged but also unique, and the equations given above still stand. Furthermore, because the exchange is slower than the overall molecular tumbling, the averaging is truly over $\left\langle r^{-6}\right\rangle$, and not $\left\langle r^{-3}\right\rangle .^{52}$ The distances and buildup slopes calculated from eqs $1-6$ are listed in Table S3.

The buildup curves were calculated with a linear regression model and the errors with an error propagation model using $\mathrm{R}[\mathrm{R}$ Core Team (2016) R: A language and environment for statistical computing (https://www.R-project.org/)].

The NMR data were processed with either NMRPipe ${ }^{53}$ or Bruker Tospsin and MestreNova. Structural data were analyzed with Bruker Topspin or MestreNova, and dynamic data were analyzed with RELAX NMR. ${ }^{54}$ 
Molecular Dynamics Simulations. Starting PDB structures of dimeric assemblies with Asn-at- $\boldsymbol{a}$ and Asn-at- $\boldsymbol{d}$ derived from PDB entry 4DZM were capped at the $\mathrm{N}$-terminus with an acetyl residue and at the $\mathrm{C}$-terminus with an amide residue. These were converted to GROMACS coordinate and topology files using the AMBER99sb-ildn force field and solvated in a dodecahedral box that is $1.5 \mathrm{~nm}$ larger than the peptide assembly in all directions with TIP3P water molecules. To simulate isotonic ion concentrations, the genion utility in GROMACS was used to add $\mathrm{Na}^{+}$and $\mathrm{Cl}^{-}$ions to a final ionic strength of $150 \mathrm{mM}$ at neutral $\mathrm{pH}$ with no overall charge. Structures were subjected to an initial 200-step energy minimization using the steepest descent method. Simulations were performed using periodic boundary conditions. Short-range electrostatic and van der Waals interactions were truncated at $1.4 \mathrm{~nm}$; long-range electrostatics were treated with the particle-mesh Ewald method, and a long-range dispersion correction was applied. The pressure was controlled by Berendsen's thermostat and the temperature by the V-rescale thermostat. Simulations were integrated with a leapfrog algorithm over a 2 fs time step, constraining bond vibrations with the P-LINCS method. For replica exchange, 64 replicas were created with exponentially distributed temperatures from 298 to $419 \mathrm{~K}$. These were subjected to an additional 200step energy minimization as before, followed by 200 ps of position-restrained $\mathrm{MD}$ in which the peptide atom coordinates were fixed. An initial 200 ps unrestrained REMD run showed average exchange rates for both simulations ranging from 0.2 to 0.3; this was judged to be sufficient for longer REMD simulation runs of $100 \mathrm{~ns}$, giving a total REMD simulation time of $6.4 \mu \mathrm{s}$ for each system. Simulations were performed using 64 nodes on the Bristol High Performance Computer BlueCrystal. ${ }^{55}$ To test for convergence of the system, the distributions of root-mean-square (rms) distance for each structure in each replica were compared and found to be equivalent (Figure S9).

Replica trajectories were concatenated such that each trajectory had a continuous temperature, and the replica with the temperature closest to that at which the NMR experiments were performed $(313 \mathrm{~K})$ was selected for analysis.

\section{RESULTS}

Multiple Asparagine Conformers Occur in Coiled-Coil Interfaces. The X-ray crystal structure of the de novo-designed parallel homodimeric CC, CC-Di [PDB entry 4DZM (Table $\mathrm{S} 1)]$, reveals a near-C2-symmetric quaternary assembly. ${ }^{17}$ However, the crystallographic unit cell contains two peptide monomers from different assemblies, and the $C 2$ symmetry is broken by the Asn residues at position 17, which populate three primary side-chain dihedrals $\left(\chi_{1}\right)$. [As detailed below, the full description of the side-chain conformation requires this and the second dihedral angle $\left(\chi_{2}\right)$, but for the immediate discussion, we need to consider only $\chi_{1}$ as this directs the side chain relative to the interface.] We refer to the three conformers as "inside" $\left(\chi_{1} \approx\right.$ $-70^{\circ}$, " $\left.g^{-"}\right)$, "middle" $\left(\chi_{1} \approx \pm 180^{\circ}\right.$, " $t$ "), and "out" $\left(\chi_{1} \approx-120^{\circ}\right.$, " $t *$ ") (Figure 1A). As a result, there are nine potential paired conformations for the Asn-Asn pair. Two of these could result in hydrogen bonding between the side chains (both "insidemiddle" conformations) if the second dihedral allowed; in six, the residues are too distant to form hydrogen bonds (the two "inside-out", "middle-middle", two "middle-out", and "outout" conformations), and there is one disallowed conformation ("inside-inside") as the Asn side chains would clash in full quaternary models.
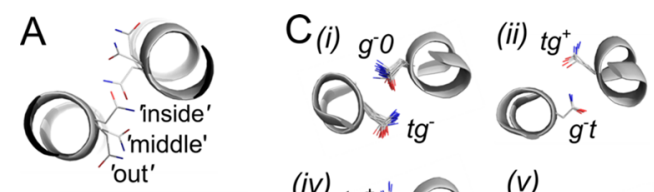

(iii) $g$

B
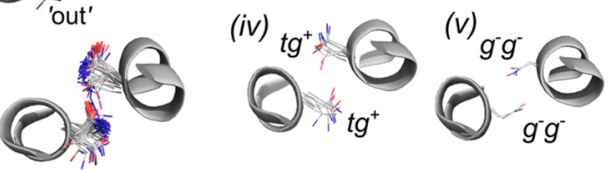

(vi)

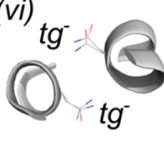

Figure 1. Conformations adopted by Asn pairs in dimeric CC interfaces of the PDB. Structures were superposed on the backbone atoms using ProFit. ${ }^{57}$ Images were generated using PyMOL (www.pymol.org). (A) Multiple conformations of Asn residues in PDB entry 4DZM. Two of the nine potential conformations allow hydrogen bonding between the Asn residues; one would be prohibited due to steric clashes, and others would point both residues out into the solvent. (B) All Asn residues at $\boldsymbol{a}$ pairs identified by $\mathrm{CC}+$ using a redundancy cutoff of $70 \%$. (C) Structures grouped according to side-chain dihedral combinations. Sidechain dihedrals $\chi_{1}$ and $\chi_{2}$ were calculated using a Python script. $\chi_{1}$ is the dihedral formed by atoms $\mathrm{N}, \mathrm{C}_{\alpha}, \mathrm{C}_{\beta}$, and $\mathrm{C}_{\gamma}$, and $\chi_{2}$ is the dihedral formed by atoms $\mathrm{C}_{\alpha}, \mathrm{C}_{\beta}, \mathrm{C}_{\gamma}$, and $\mathrm{O}_{\delta 1}$. These were classified as follows: $t$, $\chi>120^{\circ}$ or $\chi<-120^{\circ}$, with $t^{*}$ assigned a subclass of $t$ where $\chi \approx \pm 120^{\circ}$; $g^{-}, \chi<0^{\circ}$ and $\chi>-120^{\circ} ; g^{+}, \chi>0^{\circ}$ and $\chi<120^{\circ}$. ' 0 ' indicates a dihedral angle with a mean of around $0^{\circ}$, which is classified as neither $g^{-}$nor $g^{+}$: (i) 47 examples, (ii) 4 examples, (iii) 7 examples, (iv) 7 examples, (v) 2 examples, and (vi) 2 examples. Three additional combinations of sidechain dihedral angles had one example each and are not depicted: $g^{+} g^{+}$/ $g^{-} g^{+}, g^{+} g^{-} / \operatorname{tg}^{+}$, and $g^{-} g^{+} / g^{+} g^{+}$.

To test if this heterogeneity of Asn-Asn interactions was representative of Asn-containing CC dimers more generally, we inspected such structures in the RCSB PDB. ${ }^{15}$ We used CC+ ${ }^{16}$ to mine the PDB for parallel CC dimers containing pairs of Asn residues at $\boldsymbol{a}$ positions. The conformations of the Asn side chains varied (Figure 1B). However, clustering the structures according to both side-chain dihedral angles, $\chi_{1}$ and $\chi_{2}$ (mentioned above), revealed a number of specific conformations (Figure $1 \mathrm{C}$ ). The most populated conformer was the "inside-middle" arrangement, more formally $\mathrm{g}^{-} \mathrm{0} / \mathrm{tg}^{-56}$, with approximately two-thirds of structures accounting for this state [Figure $1 \mathrm{C}(\mathrm{i})]$. This promotes a buried hydrogen bond between the two Asn side chains. Of the other, less populated conformations, only one allows a hydrogen bond [Figure $1 \mathrm{C}(\mathrm{ii}), \mathrm{tg}^{+} / \mathrm{g}^{-} t$ ], which is an alternative configuration within the "inside-middle" group, equivalent to $g^{-} 0 / \mathrm{tg}^{-}$but with the amide atoms swapped. The rest either preclude a hydrogen bond altogether [Figure $1 \mathrm{C}(\mathrm{v})$ and Figure $1 \mathrm{C}(\mathrm{vi})]$ or allow a hydrogen bond only if the side chain of one Asn residue were to flip at the $\mathrm{C} \gamma$ position [Figure $1 \mathrm{C}$ (iii) and Figure $1 \mathrm{C}(\mathrm{iv})]$. This analysis assumes that the atoms have been built into these structures correctly, and given the nature of the Asn side chain, there could be some ambiguity in the placement of atoms.

As with CC-Di, we found several of the structures in the broader PDB with Asn in multiple conformations. One was in a CC from Mycobacterium tuberculosis in which three conformers of the Asn are seen on each strand (PDB entry $3 \mathrm{~m} 91^{58}$ ). This structure is complicated further by two flanking helices that provide additional Asn residues that compete for hydrogen bonding with the central Asn side chains (Figure S1). Interestingly and more generally, such potential interactions between Asn residues often became apparent only when symmetry mates were generated, and not all the combinations of conformations listed in the PDB file would be allowed due to steric clashing (Figure 1A). 
It is clear from this analysis that there are multiple possible individual and paired conformations for buried Asn residues at dimeric CC interfaces, which opens possibilities for dynamics between these.

The Interfacial Asparagine of CC-Di Is Dynamic but Forms Hydrogen Bonds. We turned to NMR spectroscopy of $\mathrm{CC}-\mathrm{Di}$ in solution to probe side-chain dynamics experimentally (Table S1 and Figure S2). ${ }^{1} \mathrm{H}$ chemical shifts for CC-Di were assigned using 2D ${ }^{1} \mathrm{H}-{ }^{1} \mathrm{H}$ correlation spectra [TOCSY and NOESY (Table S2)]. This gave a single set of resonances consistent with the largely C2-symmetric X-ray structure. We extracted structural information for CC-Di from the intramolecular connectivities observed in the $2 \mathrm{D}$ NOESY spectrum (Figure $2 \mathrm{~A}) \cdot d_{\mathrm{N}, \mathrm{N}}(i, i+1), \mathrm{d}_{\alpha, \mathrm{N}}(i, i+3)$, and $\mathrm{d}_{\alpha, \mathrm{N}}(i, i+4)$ NOEs were found along the whole peptide sequence, indicative of a fully $\alpha$-helical structure. This was corroborated by negative differences in chemical shifts ( $\Delta \delta$ values) of the $\alpha \mathrm{H}$ protons from those expected for the fully unfolded peptide (Figure $2 \mathrm{~B}$ ).


Figure 2. Structural elucidation of CC-Di in solution by NMR spectroscopy. (A) NOE connectivity table based on TOCSY and NOESY experiments for CC-Di measured at $20^{\circ} \mathrm{C}$. (B) $\Delta \delta$ values of the $\mathrm{H} \alpha$ proton chemical shifts. $\Delta \delta$ values were calculated as the difference in the observed chemical shifts and the sequence-corrected random-coil chemical shifts. ${ }^{59}$ (C) TOCSY (i), ${ }^{15} \mathrm{~N}$-edited SOFAST-HMQC (ii), and NOESY (iii) spectra showing signal broadening of the N17 amide backbone resonances ( $\mathrm{i}$ and ii) and the amide side-chain resonances (iii). Conditions: $2 \mathrm{mM}$ total peptide, $10 \mathrm{mM}$ phosphate buffer $[68 \mathrm{mM}$ sodium chloride and $1.4 \mathrm{mM}$ potassium chloride ( $\mathrm{pH}$ adjusted to 7.0)], $10 \% \mathrm{D}_{2} \mathrm{O}$, using a cryoprobe-equipped Bruker $600 \mathrm{MHz}$ spectrometer.
More specifically, we found that the Asn resonances were broadened in all NMR spectra [Figure $2 \mathrm{C}(\mathrm{i}-\mathrm{iii})]$ and that this was most apparent for the side-chain resonances [Figure $2 \mathrm{C}($ iii) $)$. This could be due in part to hydrogen bonding or even exchange with solvent. However, the cross-peak between the side-chain amide protons [Asn17(HD21-HD22)] was broad and asymmetric, indicative of multiple resonances and an ensemble of conformers [Figure 2C(iii)]. Consequently, the resulting weaker signal meant that Asn side-chain resonances were not detected in $2 \mathrm{D}{ }^{1} \mathrm{H}-{ }^{15} \mathrm{~N}$ correlation spectra $\left[{ }^{15} \mathrm{~N}\right.$-edited SOFAST-HMQC and ${ }^{1} \mathrm{H}-{ }^{15} \mathrm{~N}$ HSQC (Figure S6B)].

To resolve these issues, we introduced site-specific isotopic labeling. First, CC-Di was made with Asn $17{ }^{15} \mathrm{~N}$-labeled [CC$\mathrm{Di}_{\mathrm{N}}$ (Asn) (Table $\mathrm{S} 1$ and Figure S3)]. This gave visible Asn 17(HD) resonances in the $2 \mathrm{D}{ }^{1} \mathrm{H}-{ }^{15} \mathrm{~N}$ correlation spectrum at $20^{\circ} \mathrm{C}$. Peak broadening was observed for both resonances, but to different extents (Figure S6C): the intensity of the 17Asn(HD22) resonance was significantly decreased compared with that of $17 \mathrm{Asn}(\mathrm{HD} 21)$. On this basis, we posit that HD22 is likely involved in hydrogen bonding and, on the basis of our analysis of the $\mathrm{PDB}$, that this hydrogen bond is formed between the Asn residues of partnering CC strands.

Non-Hydrogen-Bonding Conformations Are Also Populated. At micromolar concentrations, CC-Di has a midpoint melting temperature of $78^{\circ} \mathrm{C}$ and its thermal unfolding transition starts above $40{ }^{\circ} \mathrm{C} .{ }^{17}$ This stability allowed us to investigate the temperature dependence of signal broadening and thereby to probe side-chain dynamics further, through threedimensional (3D) ${ }^{15} \mathrm{~N}$-edited NOESY at 10,20 , and $40{ }^{\circ} \mathrm{C}$ for CC-Di ${ }_{N}\left(\right.$ Asn). In the ${ }^{1} \mathrm{H}-{ }^{1} \mathrm{H}$ planes of these spectra (Figure $3 \mathrm{~A}$ ), peaks became sharper and more resonances appeared for Asn 17 as the temperature was increased. By $40{ }^{\circ} \mathrm{C}$, all peaks were sharpened and the HD22 resonances were visible. We used this $40{ }^{\circ} \mathrm{C}$ spectrum to assign NOE contacts to the Asn side-chain amide protons. The most intensive NOEs were to leucine (Leu) side chains, Leu13 or Leu20, which flank Asn17 in the hydrophobic core. This indicates strongly that the Asn side chains are buried in the hydrophobic core as expected. However, this experiment has a caveat: it does not distinguish intra- and interstrand NOEs.

To address this, we made a third CC-Di peptide variant, CC$\mathrm{Di}_{\mathrm{C}}$ (Leu13), with Leu13 labeled with ${ }^{13} \mathrm{C}$ at the $\mathrm{C} \alpha$ position (Table S1 and Figure S4), and mixed this with CC-Di ${ }_{\mathrm{N}}($ Asn) for $4 \mathrm{D}$ NOE experiments ( $\left.{ }^{13} \mathrm{C}-\mathrm{HSQC}-\mathrm{NOESY}-{ }^{15} \mathrm{~N}-\mathrm{HMQC}\right) .{ }^{60}$ The peptides were mixed in a 1:1 ratio, which, all other things being equal, should have given $50 \%$ heterolabeled dimers. Consistent with this, the $\left({ }^{1} \mathrm{H},{ }^{1} \mathrm{H}\right)$ plane from the $4 \mathrm{D}$ experiment revealed NOEs between all three nitrogen-bound protons of Asn 17 and the $\mathrm{C} \alpha$ proton of Leu13 (Figure 3B). This is possible only if the two labeled peptides are complexed and the Asn side chains spend at least some time buried in the interface. Moreover, the NOEs from Leu 13 had different intensities: the weakest was to the backbone $\mathrm{NH}$ of Asn 17, that for the HD22 proton was also weak but slightly more intensive, and the strongest was for HD21. This suggests decreasing contact distances between these pairs of protons, again consistent with at least partial burial of the Asn side chains.

We estimated the Leu13-Asn17(HD21/HD22) interproton distances relative to the Leu13-Asn17(HN) distance through an interpeptide NOE buildup experiment with mixing times of 40$120 \mathrm{~ms}$ (Figure 3C). This gave cross-relaxation rate constants for $\mathrm{HN}, \mathrm{HD} 21$, and HD22. From the X-ray crystal structure of CC$\mathrm{Di}$, the Leu13(C $\alpha \mathrm{H})-\operatorname{Asn} 17(\mathrm{NH})$ distance is $7.5 \AA$ (Figure S7). 

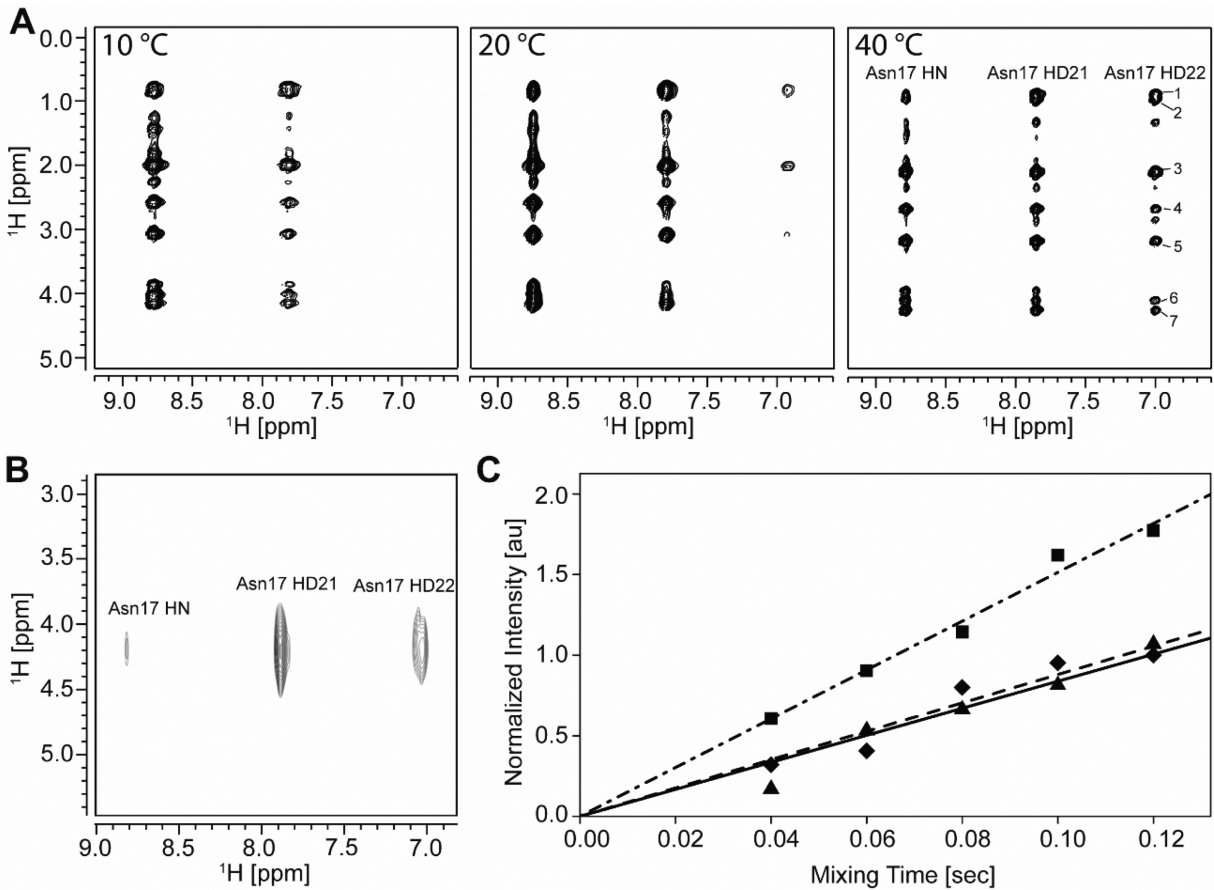

Figure 3. Multidimensional NOE experiments to estimate interchain side-chain contacts. (A) ${ }^{15} \mathrm{~N}$-edited NOESY at 10,20 , and $40{ }^{\circ} \mathrm{C}$. Assignments: 1 , HD1 Leu13/Leu20; 2, HD2 Leu13/Leu20; 3, $\beta \mathrm{H}$ Leu13/Leu20; 4/5, $\beta \mathrm{H}$ Asn 17; 6, $\alpha \mathrm{H}$ Leu13; 7, $\alpha \mathrm{H}$ Asn17/Leu20. (B) $2 \mathrm{D}$ plane of the ${ }^{13} \mathrm{C}-\mathrm{HSQC}-$ NOESY- ${ }^{15} \mathrm{~N}-\mathrm{HMQC}$ showing cross-peaks between Asn17 and Leu13 of the partnering peptides. (C) Relative intensity plot of Asn17(HN) ( $\bullet$, dashed line), HD21 ( $\boldsymbol{\square}$, dashed-dotted line), and HD22 ( $\boldsymbol{\Delta}$, solid line) as a function of mixing time in ${ }^{13} \mathrm{C}-\mathrm{HSQC}-\mathrm{NOESY}-{ }^{15} \mathrm{~N}-\mathrm{HMQC} 1 \mathrm{D}$ spectra. Intensities were normalized to Asn $17(\mathrm{HN})$. Conditions: $2 \mathrm{mM}$ total peptide, $10 \mathrm{mM}$ phosphate buffer [ $68 \mathrm{mM}$ sodium chloride and $1.4 \mathrm{mM}$ potassium chloride ( $\mathrm{pH}$ adjusted to 7.0$)], 10 \% \mathrm{D}_{2} \mathrm{O}$, using a cryoprobe-equipped Bruker $600 \mathrm{MHz}$ spectrometer (A and C) or an $800 \mathrm{MHz}$ spectrometer (B).
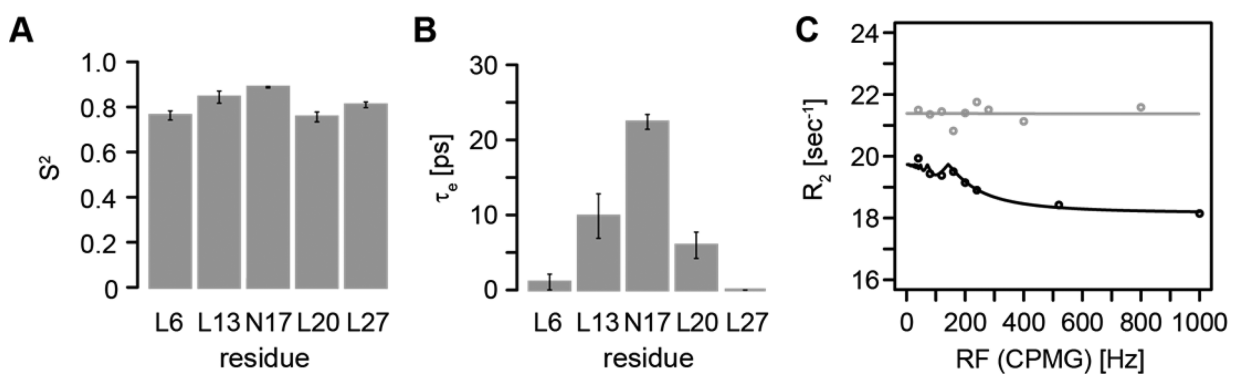

Figure 4. Dynamics in CC-Di investigated by NMR experiments. (A) $S^{2}$ and (B) $\tau_{\mathrm{e}}$ values obtained from model-free analyses for backbone amide protons of Leu6, Leu13, Asn17, Leu20, and Leu27. An $S^{2}$ of 1.0 indicates the greatest restriction of bond-vector motion. (C) Relaxation-dispersion curves for Asn 17(HD21) at $600 \mathrm{MHz}$ (black) and $700 \mathrm{MHz}$ (gray). The plots were obtained from a series of CPMG sequence experiments with varying refocusing frequencies [RF (CPMG)]. The intensity of the data at each of the refocusing frequencies was converted into an $R_{2}$ value and the resulting curve fitted to the Bloch-McConnell equations to extract the exchange rate $\left(R_{\mathrm{ex}}\right) .{ }^{55}$ The line is the fit of the selected model: "CR72 Full" slow exchange giving an $R_{\mathrm{ex}}$ of $20 \pm 4 \mathrm{~s}^{-1}$. Conditions: $1 \mathrm{mM}$ total peptide, $10 \mathrm{mM}$ phosphate buffer [ $68 \mathrm{mM}$ sodium chloride and $1.4 \mathrm{mM}$ potassium chloride (pH adjusted to 7.0$)$ ], $50 \% \mathrm{D}_{2} \mathrm{O}$, using a cryoprobe-equipped Bruker 600 and $700 \mathrm{MHz}$ spectrometer with ${ }^{2} \mathrm{H}$ decoupling.

As molecular dynamics simulations (vide infra) suggested that the backbone structure of the dimer is relatively rigid, we used this as a reference to estimate the other distances from Leu13(C $\alpha \mathrm{H})$ to Asn17(HD21/HD22) (Materials and Methods and Table S3). In this way, we measured the Leu13 $(\mathrm{C} \alpha \mathrm{H})-$ Asn17(HD21) and -Asn17(HD22) distances both as $\approx 7 \AA$, which we recognize are averages over the experimental time. From the X-ray crystal structure and MD simulations of CC-Di, with both Asn side chains buried (i.e., "inside" or "middle" conformations), these distances are expected to be 3.3-6.3 (Figure S7A-D) and if they adopt "out" conformations 6.5-7.6 $\AA$ (Figure S7E). The fact that the experimentally determined distances are toward the higher ends of these ranges indicates strongly that the non-hydrogen-bonding "out" conformations do exist in solution in a significant population, together with "inside" and "middle" conformations. However, because many conformations are potentially present, each with different distances resulting in different NOE enhancements, it is not possible to infer their relative populations.

Asparagine Conformers Exchange on the Millisecond Time Scale. To probe the dynamics of Asn17 in the dimer, we performed two relaxation NMR experiments with a peptide, CC$\mathrm{Di}_{\mathrm{N}}(\mathrm{Leu}, \mathrm{Asn})$, in which the four Leu residues at $\boldsymbol{d}$ positions and Asn 17 were fully labeled with ${ }^{15} \mathrm{~N}$ (Table S1 and Figure S5).

First, model-free analyses were performed for backbone amide protons. These probe fast motions on the picosecond to nanosecond time scale. This is done by transforming observable relaxation parameters, $T_{1}, T_{2}$, and heteronuclear NOEs (Table 
A

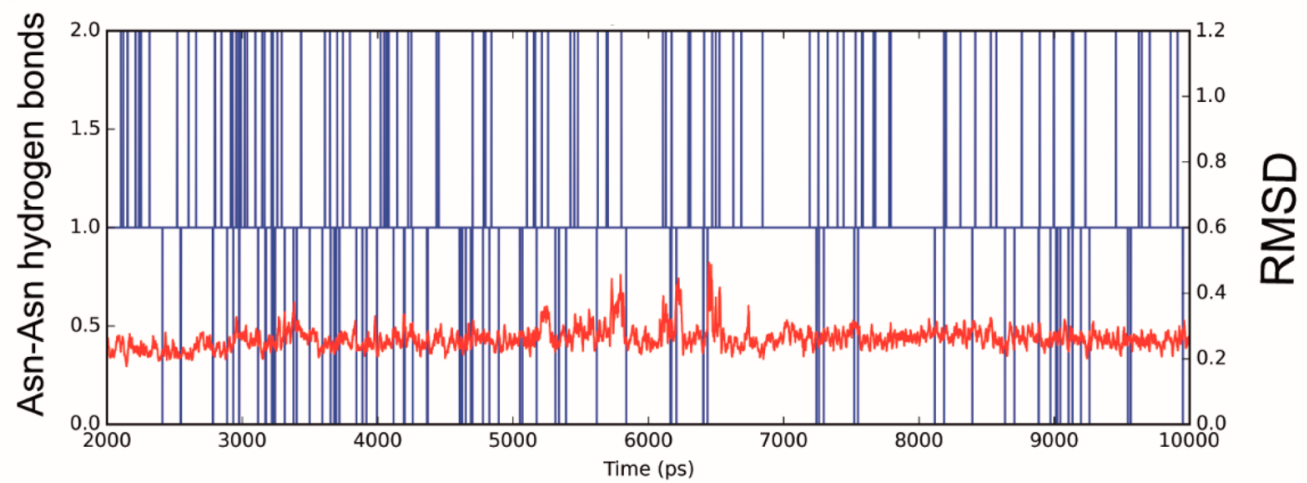

B



C
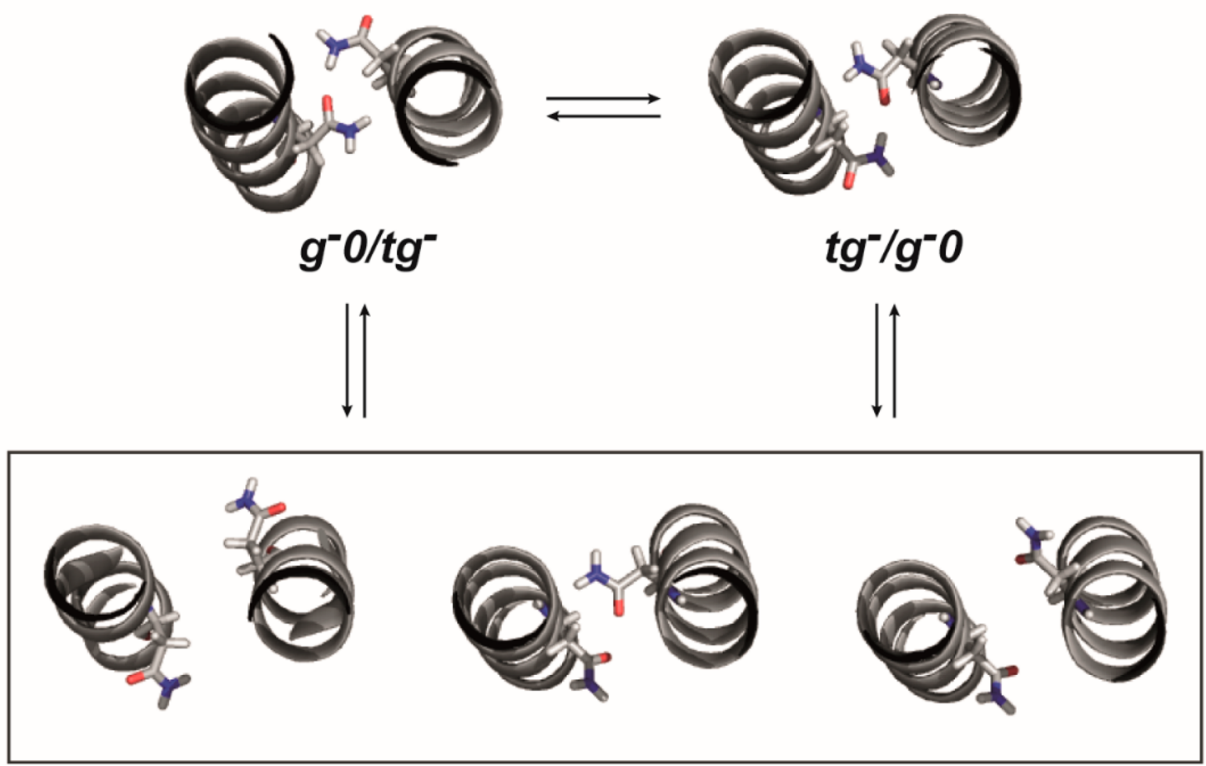

Figure 5. Dynamic equilibria of the Asn side-chain conformations. (A and B) The last $8000 \mathrm{ps}$ of the molecular dynamics trajectories at $313 \mathrm{~K}$ for the (A) Asn-at- $\boldsymbol{a}$ and (B) Asn-at- $\boldsymbol{d}$ systems described in the text. Full details of the REMD protocol can be found in the Supporting Information. The number of hydrogen bonds made between the two Asn residues in the core is colored blue, and the backbone RMSD is colored red. The Asn-at- $\boldsymbol{a}$ simulation showed continual interchange between side-chain conformations. By contrast, there were very few hydrogen bonds made between Asn-at- $\boldsymbol{d}$, and where they did occur, they were short-lived. (C) Proposed model of the dynamic processes of the Asn pair in CC-Di.

S4), into interpretable parameters: the squared generalized order parameter $\left(S^{2}\right)$ and the effective correlation time $\left(\tau_{\mathrm{e}}\right),{ }^{48}$ which indicate the amplitude and the time scale of local motions, respectively. We employed the method of d'Auvergne and Gooley, which refines the model-free coefficients using an independent tumbling time $\left(\tau_{\mathrm{m}}\right)$ for each residue and a selection of diffusion models to compute $S^{2}$ and $\tau_{\mathrm{e}}$ (RELAX). ${ }^{54}$ The $S^{2}$ values for all of the backbone amide protons were closely similar at $\approx 0.8$ (Figure $4 \mathrm{~A}$ and Table S5). This revealed that the majority of the backbone structure of the peptide was almost evenly rigid. 
However, notably, the $S^{2}$ value for Asn 17 was the largest among those of five labeled residues, indicating that the motion of the Asn 17- NH bond was the most restricted. Also, its $\tau_{\mathrm{e}}$ was the largest, by a factor of 2 compared with those of the neighboring leucines. These experiments and analyses illustrate that Asn17 has unique internal motions compared to the more rigidly held leucines in the dimer interface.

Second, to investigate conformational exchange of the Asn 17 side chain, we performed relaxation-dispersion experiments. ${ }^{61,62}$ These detect slow chemical exchange in the microsecond to millisecond regime via a series of CarrPurcell-Meiboom-Gill (CPMG) sequence experiments with varying refocusing pulse delays (Figure $4 \mathrm{C}$ ). The data indicated that the Asn 17 side-chain conformers exchange in the 10-100 ms regime, while none of backbone amide protons exhibits any comparable exchange (Figure S8). The exchange rate for HD21 was calculated more precisely at $20 \pm 4 \mathrm{~s}^{-1}$. However, because of the broader line shape of HD22, no exchange model could be reasonably fitted. This calculated value likely combines exchanges among several conformers at several exchange times and motion on a shorter time scale. Nonetheless, considering that the results of the NOE experiments indicated that the main Asn 17 side-chain conformers were with an "out" conformation, it is likely that these will experience an exchange regime in the range of $10-100 \mathrm{~ms}$.

Asparagines at $a$ Form Hydrogen Bonds That Exchange on the Picosecond to Nanosecond Time Frame. To explore Asn conformations within CC interfaces and to examine how these might influence oligomeric-state selection, we performed replica exchange molecular dynamics (REMD) simulations. ${ }^{62}$ Starting structures were generated from the X-ray crystal structure of CC-Di. ${ }^{17}$ From possible starting conformations for the Asn 17 pair (Figure 1A), we selected that with both Asn side-chain amide groups pointing "out" toward solvent. We chose this to test if a hydrogen bond would form between the Asn side chains during the simulation, rather than starting with a lower-energy hydrogen-bonded state and attempting to see bond rupture. Indeed, we found that hydrogen bonding between the Asn side chains appeared $\approx 200$ ps into the simulation. This became the default state around $5 \mathrm{~ns}$, and the structure remained stable from that point on (Figure 5A). Nonetheless, the hydrogen bond was very dynamic. The Asn residues switched frequently between two forms of one conformation, with concomitant breaking and re-forming of the hydrogen bonds (Figure S9C). The observed conformation matched the most popular hydrogen-bonded conformation from the PDB analysis [Figure $1 \mathrm{C}(\mathrm{i})$ ], i.e., $\mathrm{g}^{-} \mathrm{O} / \mathrm{tg}^{-}$or "insidemiddle". What was not obvious from the latter static structures, but became apparent in the REMD, was that the Asn residues interconvert between the two equivalent conformations, $g^{-} \mathrm{O} / \mathrm{tg}^{-}$ and $\mathrm{tg}^{-} / \mathrm{g}^{-} \mathrm{O}$. Effectively, the side chains of the two peptides switch conformations and, therefore, exchange between being a hydrogen-bond acceptor and a donor (Figure 5C). This interconversion occurred $\sim 2800$ times in $\sim 7800$ consecutive snapshots of the simulation that had exactly one hydrogen bond between the two residues; i.e., the hydrogen bond was retained between steps, but the conformation of the asparagine pair switched.

Thus, pairs of Asn residues at position $\boldsymbol{a}$ are accommodated in the otherwise hydrophobic interfaces of parallel CC dimers, and some of the loss of solvation energy is compensated by the formation of interhelix side-chain interactions. However, these hydrogen bonds are not static but extremely dynamic, with different rotamers of the Asn being explored to effect exchange between outward- and inward-facing amide groups, with only the latter leading to hydrogen bonding.

For comparison, a second REMD simulation was performed with Asn residues at a $\boldsymbol{d}$ position of CC-Di. For this, a starting structure was constructed from 4DZM in which position 17 was mutated to Ile, to match the other $a$ positions in the structure, and position 13 was mutated to Asn. Asn rotamers were chosen on the basis of the small number of CC dimers found in CC+ with Asn at position $d .{ }^{16}$ In contrast to the Asn-at-a simulation, the second calculation revealed that Asn residues paired at $\boldsymbol{d}$ positions rarely form a hydrogen bond (Figure 5B). Moreover, on the odd occasion that a hydrogen bond was made, it was at the expense of the overall structure, a kink formed in the helix near the Asn position (Figure S9D); this destabilizes the core, and the helical structure unfolds C-terminal to the Asn residues. Consistent with this, we found that Asn occurs only rarely at $d$ positions in dimeric CC structures: there were 19 examples in $\mathrm{CC}+$ at $50 \%$ redundancy, compared with 128 examples of Asn at $a$ sites. ${ }^{17}$ Interestingly, the majority of the former also have an Asn at position $\boldsymbol{a}$, which may help accommodate an Asn at position $d$ by specifying the dimer state. These findings are consistent with recently described experimental systems that show that Asn at position $\boldsymbol{d}$ destabilizes dimers more than Asn at position $\boldsymbol{a}$, and that Asn at position $\boldsymbol{d}$ is better accommodated in parallel trimeric CCs. ${ }^{17,24,32}$

\section{DISCUSSION}

The coiled-coil (CC) motif is one of the most widely used peptide building blocks in synthetic biology and biomaterials. ${ }^{2,3}$ Such work relies on having sequence-structure relationships to guide and deliver peptide designs de novo. Ideally, these relationships should be understood at the physicochemical level. For CCs, such rules do exist, but the underlying mechanisms of action are not understood for all of these. Here we focus on examining in detail one such rule of thumb, namely, that asparagine (Asn) residues placed at the $\boldsymbol{a}$ sites of the heptad sequences repeats of CCs, abcdefg, direct the formation of parallel dimeric CCs. These positions are traditionally considered as being buried within the otherwise hydrophobic CC interface, and as such, they are usually paired with another Asn residue in the partnering strand of the CC. It is assumed in the literature that specificity for parallel, in-register dimers is conferred by the abilities of these Asn pairs to form inter-sidechain hydrogen bonds.

Our study combines bioinformatics analysis of known X-ray crystal structures of dimeric CCs to explore the static conformations accessible to these buried Asn residues and NMR experiments and MD simulations to probe the dynamic states accessible to these pairings. The CC crystal structures show different conformations for Asn where the side chain points "inside", toward the "middle", and "outside" of the CC interface. Only certain combinations have the potential to make side-chain hydrogen bonds, however, namely, "inside-middle". Although other paired conformers have been observed, this is by far the dominant one.

2D NMR experiments of a designed CC dimer with an Asn-at$\boldsymbol{a}$ (CC-Di) confirm a fully $\alpha$-helical structure but, importantly, reveal significant broadening of both the Asn backbone and sidechain resonances indicative of chemical exchange. Intra- and interstrand NOE contacts with the Asn residues are obtained from 3D and 4D NMR experiments with ${ }^{15} \mathrm{~N}$-labeled peptides. These confirm contacts to neighboring leucine residues of the 
hydrophobic core but show reduced-intensity cross-peaks indicating that the Asn side chains are not exclusively inside the core. Moreover, many of the possible combinations of "inside", "middle", and "out" conformations appear to be populated in an ensemble of local structures. These dynamics are probed further with model-free analysis and relaxationdispersion experiments. The former confirm that the backbone structure of CC-Di is evenly rigid, but with unique internal motions for Asn17, and the latter reveal millisecond chemical exchange of its side-chain amide protons. Together, these data strongly suggest that the Asn 17 side chain is in conformational exchange on a $10-100 \mathrm{~ms}$ time scale. MD studies of CC-Di, initiated with a non-hydrogen-bonded "inside-inside" pair conformation, confirm the formation of the "inside-middle" hydrogen bond, and that this is a highly dynamic state switching between two analogous side-chain dihedral conformations. By contrast, MD simulations of a CC-Di variant with Asn-at- $d$ show that the side chains are unable to form hydrogen-bonded pairs in a parallel CC dimer. This is in accord with the relatively few examples of CCs with Asn-at-d, ${ }^{16,63-65}$ and with a recent experimental study in which the Leu $13 \rightarrow$ Asn mutation of CCDi eliminates dimer specificity. ${ }^{32}$ This underlines the importance and worth of Asn-at- $\boldsymbol{a}$ as a protein design rule.

Taking all of these results into account, we propose the following model (Figure 5C). Asn residues at the $\boldsymbol{a}$ positions of parallel coiled-coil dimers increase the dimer specificity because the formation of a hydrogen bond between side-chain amide groups is generally enabled. However, the Asn pair can adopt several conformations in a dynamic equilibrium. The most preferred conformations are the two "inside-middle" pairs, $g^{-} 0$ / $\mathrm{tg}^{-}$and $\mathrm{tg}^{-} / \mathrm{g}^{-} \mathrm{O}$. These both lead to the formation of hydrogen bonds between the Asn side chains, and there is fast exchange between the two conformations. This constant bond breaking and making occasionally results in a switch from an "insidemiddle" conformation to "out" conformations, most likely driven by the hydrophilicity of the Asn residue. Subsequent hydrogenbond formation is potentially slowed because the "out" conformations can be stabilized by contacts with polar groups and water. This chemical exchange in the millisecond regime is likely to be responsible for signal broadening of the asparagine resonances in the NMR experiments.

Our model has features in common with that from a foregoing study of NMR studies of the Jun leucine zipper, which is another CC dimer. ${ }^{46}$ However, we have been able to address details and specifics of the mechanism to an unprecedented level by identifying the different conformational states that are possible through analyses of many X-ray crystal structures; quantifying the time scales of the dynamics and exchange processes with multidimensional NMR experiments; validating a sequential mechanism for these processes using MD simulations; and comparing data for how different Asn inclusions specify dimer or do not. This presents a holistic view of the roles of buried Asn residues in promoting and maintaining CC-dimer states.

A recent NMR study by Kaplan et al. ${ }^{44}$ further highlights the significance of the dynamics of side chains on CC structure. As the $\mathrm{pH}$ is decreased from near neutral to acidic in the GCN4-p1 system, interchain salt bridges are lost and the titratable (glutamate and lysine) side chains involved become more dynamic. As a consequence, and although helicity and CC interactions are maintained, the whole structure relaxes, with the helices moving slightly farther apart and the supercoil unwinding slightly.
While these and other studies illustrate that dynamics in CC systems have not been entirely overlooked, it is true to say that much of knowledge of these relationships comes from examining static structures and experiments that assume near-static structures. Our study adds to this move toward a holistic view of these assemblies that considers dynamics, as well. We have focused on buried Asn residues in CC dimers and found that these are far more dynamic than previously thought with the inclusion of different Asn conformations in the hydrogenbonded state, and even that the Asn side chains temporarily shift outside of the hydrophobic core. The latter observation has not been described in any foregoing reports. This helps to explain why the incorporation of polar residues is actually destabilizing, because a favorable contribution from buried polar residues to stability is possible only if the unfavorable energy of dehydration is completely compensated by hydrogen bonding within the folded state. ${ }^{66-70}$ Understanding these effects should help in the design of alternative specification motifs for distinguishing between CC states. Indeed, this has been attempted to some extent, though without considerations of structural dynamics, using urea and guanidinium recognition motifs. ${ }^{71-73}$ Generally, incorporating dynamical aspects of CC structure, assembly, and specificity will improve our ability to model, engineer, and design these important protein-protein recognition motifs.

\section{ASSOCIATED CONTENT}

\section{S Supporting Information}

The Supporting Information is available free of charge on the ACS Publications website at DOI: 10.1021/acs.biochem.7b00848.

Materials and methods as well as additional figures and tables (PDF)

\section{AUTHOR INFORMATION}

\section{Corresponding Authors}

*E-mail: fthomas@gwdg.de.

*E-mail: d.n.woolfson@bristol.ac.uk.

ORCID $\odot$

Derek N. Woolfson: 0000-0002-0394-3202

\section{Author Contributions}

F.T., A.N., and D.N.W. designed experiments. F.T. and A.N. synthesized the peptides. F.T., A.N., and A.O. recorded and analyzed the NMR spectra. G.J.B. performed the analyses of the Protein Data Bank and MD simulations. All authors contributed to writing the manuscript. F.T., A.N., A.O., and G.J.B. contributed equally to this work.

\section{Funding}

F.T. and D.N.W. are grateful for financial support from the Leverhulme Trust (RPG-2012-536). A.N. and D.N.W. were funded by the BBSRC (BB/J009784/1). A.N., G.J.B., and D.N.W. were funded by the ERC (340764). D.N.W. holds a Royal Society Research Merit Award (WM140008). The authors appreciate the support of the MRC Biomedical NMR Centre at the Francis Crick Institute, London, which is core funded by Cancer Research UK, the UK Medical Research Council, and the Welcome Trust (FC001142 and FC10029).

\section{Notes}

The authors declare no competing financial interest. 


\section{ACKNOWLEDGMENTS}

The authors thank Tom Frenkiel, Geoff Kelly, Matt Crump, Craig Butts, Emily Baker, and Richard Sessions for fruitful discussions.

\section{REFERENCES}

(1) Lupas, A. N., and Bassler, J. (2017) Coiled Coils - A Model System for the 21st Century. Trends Biochem. Sci. 42, 130-140.

(2) Woolfson, D. N. (2017) Coiled-Coil Design: Updated and Upgraded. Subcell. Biochem. 82, 35-61.

(3) Boyle, A. L., and Woolfson, D. N. (2011) De novo designed peptides for biological applications. Chem. Soc. Rev. 40, 4295-4306.

(4) Robson Marsden, H., and Kros, A. (2010) Self-Assembly of Coiled Coils in Synthetic Biology: Inspiration and Progress. Angew. Chem., Int. Ed. 49, 2988-3005.

(5) Woolfson, D. N., Bartlett, G. J., Bruning, M., and Thomson, A. R. (2012) New currency for old rope: from coiled-coil assemblies to alphahelical barrels. Curr. Opin. Struct. Biol. 22, 432-441.

(6) Crick, F. H. C. (1953) The packing of alpha-helices - simple coiledcoils. Acta Crystallogr. 6, 689-697.

(7) Thomson, A. R., Wood, C. W., Burton, A. J., Bartlett, G. J., Sessions, R. B., Brady, R. L., and Woolfson, D. N. (2014) Computational design of water-soluble alpha-helical barrels. Science 346, 485-488.

(8) Harbury, P. B., Zhang, T., Kim, P. S., and Alber, T. (1993) A switch between 2-stranded, 3-stranded and 4-stranded coiled coils in GCN4 leucine-zipper mutants. Science 262, 1401-1407.

(9) O'Shea, E. K., Lumb, K. J., and Kim, P. S. (1993) Peptide Velcro Design of a heterodimeric coiled-coil. Curr. Biol. 3, 658-667.

(10) McClain, D. L., Gurnon, D. G., and Oakley, M. G. (2002) Importance of potential interhelical salt-bridges involving interior residues for coiled-coil stability and quaternary structure. J. Mol. Biol. $324,257-270$

(11) Steinmetz, M. O., Jelesarov, I., Matousek, W. M., Honnappa, S., Jahnke, W., Missimer, J. H., Frank, S., Alexandrescu, A. T., and Kammerer, R. A. (2007) Molecular basis of coiled-coil formation. Proc. Natl. Acad. Sci. U. S. A. 104, 7062-7067.

(12) Litowski, J. R., and Hodges, R. S. (2002) Designing heterodimeric two-stranded alpha-helical coiled-coils - Effects of hydrophobicity and alpha-helical propensity on protein folding, stability, and specificity. J. Biol. Chem. 277, 37272-37279.

(13) Thomas, F., Boyle, A. L., Burton, A. J., and Woolfson, D. N. (2013) A Set of de Novo Designed Parallel Heterodimeric Coiled Coils with Quantified Dissociation Constants in the Micromolar to Subnanomolar Regime. J. Am. Chem. Soc. 135, 5161-5166.

(14) Monera, O. D., Kay, C. M., and Hodges, R. S. (1994) Electrostatic interactions control the parallel and antiparallel orientation of alphahelical chains in two-stranded alpha-helical coiled-coils. Biochemistry 33, 3862-3871.

(15) Berman, H. M., Westbrook, J., Feng, Z., Gilliland, G., Bhat, T. N., Weissig, H., Shindyalov, I. N., and Bourne, P. E. (2000) The Protein Data Bank. Nucleic Acids Res. 28, 235-242.

(16) Testa, O. D., Moutevelis, E., and Woolfson, D. N. (2009) CC plus: a relational database of coiled-coil structures. Nucleic Acids Res. 37, D315-D322.

(17) Fletcher, J. M., Boyle, A. L., Bruning, M., Bartlett, G. J., Vincent, T. L., Zaccai, N. R., Armstrong, C. T., Bromley, E. H. C., Booth, P. J., Brady, R. L., Thomson, A. R., and Woolfson, D. N. (2012) A basis set of de novo coiled-coil peptide oligomers for rational protein design and synthetic biology. ACS Synth. Biol. 1, 240-250.

(18) Zeng, X., Herndon, A. M., and Hu, J. C. (1997) Buried asparagines determine the dimerization specificities of leucine zipper mutants. Proc. Natl. Acad. Sci. U. S. A. 94, 3673-3678.

(19) Zhu, H., Celinski, S. A., Scholtz, J. M., and Hu, J. C. (2000) The contribution of buried polar groups to the conformational stability of the GCN4 coiled coil. J. Mol. Biol. 300, 1377-1387.

(20) Oakley, M. G., and Kim, P. S. (1998) A buried polar interaction can direct the relative orientation of helices in a coiled coil. Biochemistry $37,12603-12610$.
(21) Akey, D. L., Malashkevich, V. N., and Kim, P. S. (2001) Buried polar residues in coiled-coil interfaces. Biochemistry 40, 6352-6360.

(22) Rackham, O. J. L., Madera, M., Armstrong, C. T., Vincent, T. L., Woolfson, D. N., and Gough, J. (2010) The Evolution and Structure Prediction of Coiled Coils across All Genomes. J. Mol. Biol. 403, 480493.

(23) Eckert, D. M., Malashkevich, V. N., and Kim, P. S. (1998) Crystal structure of GCN4-pIQI, a trimeric coiled coil with buried polar residues. J. Mol. Biol. 284, 859-865.

(24) Hartmann, M. D., Ridderbusch, O., Zeth, K., Albrecht, R., Testa, O., Woolfson, D. N., Sauer, G., Dunin-Horkawicz, S., Lupas, A. N., and Alvarez, B. H. (2009) A coiled-coil motif that sequesters ions to the hydrophobic core. Proc. Natl. Acad. Sci. U. S. A. 106, 16950-16955.

(25) O'Shea, E. K., Klemm, J. D., Kim, P. S., and Alber, T. (1991) X-ray structure of the GCN4 leucine zipper, a 2-stranded, parallel coiled coil. Science 254, 539-544.

(26) Glover, J. N. M., and Harrison, S. C. (1995) Crystal-structure of the heterodimeric bzip transcription factor c-Fos-c-Jun bound to DNA. Nature 373, 257-261.

(27) Lumb, K. J., and Kim, P. S. (1995) A buried polar interaction imparts structural uniqueness in a designed heterodimeric coiled coil. Biochemistry 34, 8642-8648.

(28) Gonzalez, L., Woolfson, D. N., and Alber, T. (1996) Buried polar residues and structural specificity in the GCN4 leucine zipper. Nat. Struct. Biol. 3, 1011-1018.

(29) Gradisar, H., and Jerala, R. (2011) De novo design of orthogonal peptide pairs forming parallel coiled-coil heterodimers. J. Pept. Sci. 17, 100-106.

(30) Crooks, R. O., Lathbridge, A., Panek, A. S., and Mason, J. M. (2017) Computational Prediction and Design for Creating Iteratively Larger Heterospecific Coiled Coil Sets. Biochemistry 56, 1573-1584.

(31) Drobnak, I., Gradisar, H., Ljubetic, A., Merljak, E., and Jerala, R. (2017) Modulation of Coiled-Coil Dimer Stability through Surface Residues while Preserving Pairing Specificity. J. Am. Chem. Soc. 139, 8229-8236.

(32) Fletcher, J. M., Bartlett, G. J., Boyle, A. L., Danon, J. J., Rush, L. E., Lupas, A. N., and Woolfson, D. N. (2017) N@a and N@d: Oligomer and partner specification by asparagine in coiled-coil interfaces. ACS Chem. Biol. 12, 528-538.

(33) Wu, Y. Y., and Collier, J. H. (2017) alpha-Helical coiled-coil peptide materials for biomedical applications. Wiley Interdiscip. Rev. Nanomed. Nanobiotechnol 9, e1424.

(34) Boyle, A. L., Bromley, E. H. C., Bartlett, G. J., Sessions, R. B., Sharp, T. H., Williams, C. L., Curmi, P. M. G., Forde, N. R., Linke, H., and Woolfson, D. N. (2012) Squaring the Circle in Peptide Assembly: From Fibers to Discrete Nanostructures by de Novo Design. J. Am. Chem. Soc. 134, 15457-15467.

(35) Gradisar, H., Bozic, S., Doles, T., Vengust, D., Hafner-Bratkovic, I., Mertelj, A., Webb, B., Sali, A., Klavzar, S., and Jerala, R. (2013) Design of a single-chain polypeptide tetrahedron assembled from coiled-coil segments. Nat. Chem. Biol. 9, 362-366.

(36) Boyken, S. E., Chen, Z. B., Groves, B., Langan, R. A., Oberdorfer, G., Ford, A., Gilmore, J. M., Xu, C. F., DiMaio, F., Pereira, J. H., Sankaran, B., Seelig, G., Zwart, P. H., and Baker, D. (2016) De novo design of protein homo-oligomers with modular hydrogen-bond network-mediated specificity. Science 352, 680-687.

(37) Lavigne, P., Kay, C. M., Sonnichsen, F. D., Hodges, R. S., Lumb, K. J., and Kim, P. S. (1996) Interhelical salt bridges, coiled-coil stability, and specificity of dimerization. Science 271, 1136-1138.

(38) Lumb, K. J., and Kim, P. S. (1996) Interhelical salt bridges, coiledcoil stability, and specificity of dimerization - Response. Science 271, $1137-1138$

(39) Meier, M., Stetefeld, J., and Burkhard, P. (2010) The many types of interhelical ionic interactions in coiled coils - An overview. J. Struct. Biol. 170, 192-201.

(40) Marti, D. N., and Bosshard, H. R. (2004) Inverse electrostatic effect: Electrostatic repulsion in the unfolded state stabilizes a leucine zipper. Biochemistry 43, 12436-12447. 
(41) Marti, D. N., and Bosshard, H. R. (2003) Electrostatic interactions in leucine zippers: Thermodynamic analysis of the contributions of Glu and his residues and the effect of mutating salt bridges. J. Mol. Biol. 330, 621-637.

(42) Lumb, K. J., and Kim, P. S. (1995) Measurement of interhelical electrostatic interactions in the GCN4 leucine-zipper. Science 268, 436439.

(43) Matousek, W. M., Ciani, B., Fitch, C. A., Garcia-Moreno E, B., Kammerer, R. A., and Alexandrescu, A. T. (2007) Electrostatic contributions to the stability of the GCN4 leucine zipper structure. J. Mol. Biol. 374, 206-219.

(44) Kaplan, A. R, Brady, M. R., Maciejewski, M. W., Kammerer, R. A., and Alexandrescu, A. T. (2017) Nuclear Magnetic Resonance Structures of GCN4p Are Largely Conserved When Ion Pairs Are Disrupted at Acidic $\mathrm{pH}$ but Show a Relaxation of the Coiled Coil Superhelix. Biochemistry 56, 1604-1619.

(45) Oshaben, K. M., Salari, R., McCaslin, D. R., Chong, L. T., and Horne, W. S. (2012) The Native GCN4 Leucine-Zipper Domain Does Not Uniquely Specify a Dimeric Oligomerization State. Biochemistry 51, 9581-9591.

(46) Junius, F. K., Mackay, J. P., Bubb, W. A., Jensen, S. A., Weiss, A. S., and King, G. F. (1995) Nuclear-magnetic-resonance characterization of the Jun leucine-zipper domain - unusual properties of coiled-coil interfacial polar residues. Biochemistry 34, 6164-6174.

(47) Schanda, P., and Brutscher, B. (2005) Very Fast TwoDimensional NMR Spectroscopy for Real-Time Investigation of Dynamic Events in Proteins on the Time Scale of Seconds. J. Am. Chem. Soc. 127, 8014-8015.

(48) Lipari, G., and Szabo, A. (1982) Model-free approach to the interpretation of nuclear magnetic-resonance relaxation in macromolecules. 1. Theory and range of validity. J. Am. Chem. Soc. 104, 45464559.

(49) Mulder, F. A. A., Skrynnikov, N. R., Hon, B., Dahlquist, F. W., and Kay, L. E. (2001) Measurement of slow (mu s-ms) time scale dynamics in protein side chains by $\mathrm{N}-15$ relaxation dispersion NMR spectroscopy: Application to Asn and Gln residues in a cavity mutant of T4 lysozyme. J. Am. Chem. Soc. 123, 967-975.

(50) Zuiderweg, E. R. P., Petros, A. M., Fesik, S. W., and Olejniczak, E. T. (1991) 4-Dimensional C-13, H-1, C-13, H-1 HMQC-NOE-HMQC NMR-spectroscopy - resolving tertiary NOE distance constraints in the spectra of larger proteins. J. Am. Chem. Soc. 113, 370-372.

(51) Vögeli, B. (2014) The nuclear Overhauser effect from a quantitative perspective. Prog. Nucl. Magn. Reson. Spectrosc. 78, 1-46.

(52) Neuhaus, D., and Williamson, M. P. (1989) The Nuclear Overhauser Effect in Structural and Conformational Analysis, 2nd ed., Wiley-VCH, Weinheim, Germany.

(53) Delaglio, F., Grzesiek, S., Vuister, G. W., Zhu, G., Pfeifer, J., and Bax, A. (1995) NMRPipe - a multidimensional spectral processing system based on Unix pipes. J. Biomol. NMR 6, 277-293.

(54) d'Auvergne, E. J., and Gooley, P. R. (2008) Optimisation of NMR dynamic models II. A new methodology for the dual optimization of the model-free parameters and the Brownian rotational diffusion tensor. $J$. Biomol. NMR 40, 121-133.

(55) BlueCrystal. http://www.acrc.bris.ac.uk/.

(56) Lovell, S. C., Word, J. M., Richardson, J. S., and Richardson, D. C. (1999) Asparagine and glutamine rotamers: B-factor cutoff and correction of amide flips yield distinct clustering. Proc. Natl. Acad. Sci. U. S. A. $96,400-405$.

(57) McLachlan, A. D. (1982) Rapid comparison of protein structures. Acta Crystallogr., Sect. A: Cryst. Phys., Diffr., Theor. Gen. Crystallogr. 38, 871-873.

(58) Wang, T., Darwin, K. H., and Li, H. L. (2010) Binding-induced folding of prokaryotic ubiquitin-like protein on the Mycobacterium proteasomal ATPase targets substrates for degradation. Nat. Struct. Mol. Biol. 17, 1352-U1208.

(59) Kjaergaard, M., and Poulsen, F. M. (2011) Sequence correction of random coil chemical shifts: correlation between neighbor correction factors and changes in the Ramachandran distribution. J. Biomol. NMR 50, 157-165.
(60) Kay, L. E., Clore, G. M., Bax, A., and Gronenborn, A. M. (1990) 4Dimensional heteronuclear triple-resonance NMR-spectroscopy of Interleukin-1-beta in solution. Science 249, 411-414.

(61) Liu, A. Z., Yao, L. S., Li, Y., and Yan, H. G. (2007) TROSY of sidechain amides in large proteins. J. Magn. Reson. 186, 319-326.

(62) Loria, J. P., Rance, M., and Palmer, A. G. (1999) A relaxationcompensated Carr-Purcell-Meiboom-Gill sequence for characterizing chemical exchange by NMR spectroscopy. J. Am. Chem. Soc. 121, 23312332.

(63) Murphy, G. A., Spedale, E. J., Powell, S. T., Pillus, L., Schultz, S. C., and Chen, L. (2003) The Sir4 C-terminal coiled coil is required for telomeric and mating type silencing in Saccharomyces cerevisiae. J. Mol. Biol. 334, 769-780.

(64) Frye, J., Klenchin, V. A., and Rayment, I. (2010) Structure of the tropomyosin overlap complex from chicken smooth muscle: insight into the diversity of N-terminal recognition. Biochemistry 49, 4908-4920.

(65) Qiao, R., Cabral, G., Lettman, M. M., Dammermann, A., and Dong, G. (2012) SAS-6 coiled-coil structure and interaction with SAS-5 suggest a regulatory mechanism in C. elegans centriole assembly. EMBO J. 31, 4334-4347.

(66) Kondratova, M. S., and Efimov, A. V. (2002) A systematic analysis of buried polar side chains and their interactions in alpha-helical proteins. Mol. Biol. 36, 117-123.

(67) Brazhnikov, E. V., and Efimov, A. V. (2005) Analysis of interactions of buried polar side chains in beta-proteins. Mol. Biol. 39, 878-886.

(68) Pace, C. N. (2001) Polar group burial contributes more to protein stability than nonpolar group burial. Biochemistry 40, 310-313.

(69) Takano, K., Yamagata, Y., and Yutani, K. (2001) Contribution of polar groups in the interior of a protein to the conformational stability. Biochemistry 40, 4853-4858.

(70) Loladze, V. V., Ermolenko, D. N., and Makhatadze, G. I. (2002) Thermodynamic consequences of burial of polar and non-polar amino acid residues in the protein interior. J. Mol. Biol. 320, 343-357.

(71) Diss, M. L., and Kennan, A. J. (2008) Orthogonal recognition in dimeric coiled coils via buried polar-group modulation. J. Am. Chem. Soc. 130, 1321-1327.

(72) Diss, M. L., and Kennan, A. J. (2008) Heterotrimeric Coiled Coils with Core Residue Urea Side Chains. J. Org. Chem. 73, 9752-9755.

(73) Diss, M. L., and Kennan, A. J. (2008) Simultaneous Directed Assembly of Three Distinct Heterodimeric Coiled Coils. Org. Lett. 10, $3797-3800$ 